\title{
A development of an epiduroscopy training simulator based on spatial cognition learning
}

\author{
Seong Wook Jang ${ }^{1}$, Junho Ko${ }^{2}$, Yu Jin Choi ${ }^{3}$, Yoon Sang Kim ${ }^{4}$ \\ ${ }_{1,3,4}$ Department of Computer Science and Engineering, Korea University of Technology and Education, \\ Cheonan, Republic of Korea \\ ${ }^{2,4}$ Institute for Bioengineering Application Technology, Korea University of Technology and Education, \\ Cheonan, Republic of Korea \\ ${ }^{4}$ Corresponding author \\ E-mail: ${ }^{1}$ elderlord@koreatech.ac.kr, ${ }^{2}$ lich126@koreatech.ac.kr, ${ }^{3}$ ekgkrha@koreatech.ac.kr, \\ 4yoonsang@koreatech.ac.kr
}

Abstract. An epiduroscopy is an effective minimally invasive surgery (MIS) used for chronic lumbago and lumbar disc herniation. As such an endoscopy is hard to learn high-level surgical skills, efficient surgical training method or system is required. Therefore, this paper proposed an epiduroscopy training simulator based on spatial cognition learning. The proposed training simulator provided four scenarios for catheter manipulation training, and it provides quantitative training results based on various measured data (completion time, number of violation and surgical instrument trajectory).

Keywords: spatial cognition learning, epiduroscopy, surgical simulator, training simulator.

\section{Introduction}

An epiduroscopy is a very effective minimally invasive surgery (MIS) used for chronic lumbago and lumbar disc herniation. MIS requires a fine surgery which inserts and controls a subminiature endoscope ( $1 \mathrm{~mm}$ diameter) and laser into a cavity through only 2D CT image. As an endoscopy is hard to learn high-level surgical skills, efficient surgical training method or system is required $[1,2]$.

Conventional surgical training is accomplished as a trainee performs a supportive role in the OR (operating room). Such a surgical provides limited learning opportunities. Furthermore, it is hard to learn an interaction between surgical instruments and human organs [3]. Therefore, various surgical training simulators have been developed that enable iterative learning for the interaction between surgical instruments and human organs. Most surgical training simulators developed so far have been related with endoscopy including bronchoscopy [4], laparoscopy [5], and arthroscopy [6]. The endoscopy training simulators provided a learning opportunity for trainees to experience surgery indirectly by constructing an environment virtually similar to real surgery. However, three dimensional spatial cognitive learning of the surgical instrument insertion path and surgical site in the human body was not considered.

Therefore, this paper proposes an epiduroscopy training simulator based on spatial cognition learning. Because catheter manipulation in epiduroscopy is the most important factor in determining the success of the surgery, the proposed training simulator providing various scenarios for catheter manipulation training. The catheter manipulation training scenarios improve a catheter manipulation capability inserted inside the human body including various nerve, lumbar and disc, based on spatial cognitive learning. At training completion, it provides the quantitative training result based on various measured data.

\section{Epiduroscopy training simulator based on spatial cognitive learning}

The spatial cognitive learning has been widely studied to analyze the person's pathfinding behavior in a field of psychology and geography [7]. From these studies, it is found that landmark knowledge is developed into survey knowledge through route one [8-10]. The survey knowledge 
means that information about the surrounding space that is drawn in the mind of the trainees' like a map. The spatial information needs to be organized into feature and structural information to use this survey knowledge [11]. Because the spatial cognitive learning is effective at improving the pathfinding capability [12], this paper aims to improve trainees' the catheter manipulation capability by applying to the endoscopic skills required high pathfinding capability. In this section, to apply spatial cognitive learning to epiduroscopy training, the spatial information related to the path of entry of surgical instruments, such as catheters, is classified into the landmark information and the path information. The landmark information corresponds to the reference points providing the context of the paths, and epiduroscopy relation to important human organs (lumbar, nerve and disk) surrounding the path of surgical instruments. And then, the path information corresponds to the catheter insertion path.

Fig. 1 shows the catheter insertion path of epiduroscopy applying the spatial cognitive learning. Fig. 1(a) shows the landmark information learning step in which the trainees cannot recognize the space inside the human body inserted the catheter (because the landmark information cannot be contextualized as path information). Fig. 1(b) shows the path information learning step that allows the trainee to recognize the insertion path of the catheter by contextualizing the landmark information of Fig. 1(a) into the landmark information.

During epiduroscopy training simulator based on the above two step spatial cognitive learning process, the trainees effectively learn catheter difficult manipulation by contextualizing important human organs (landmark information) surrounding the path of the surgical instrument in repeated training to path information.

The proposed epiduroscopy training simulator based on the spatial cognitive learning consists of visualization part, training part and evaluation part as shown in Fig. 2.

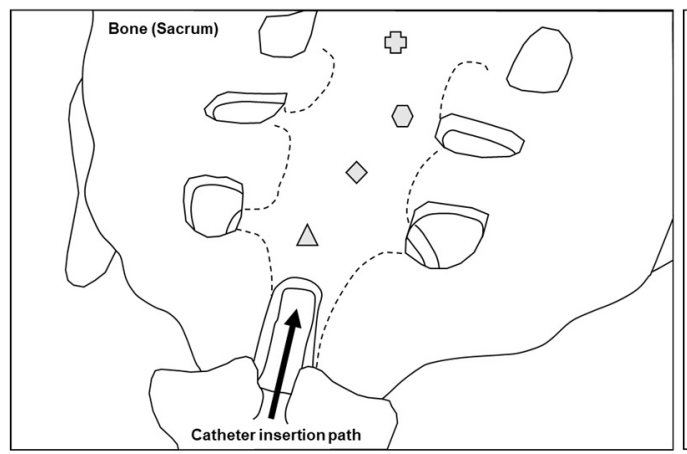

a) Landmark information learning step

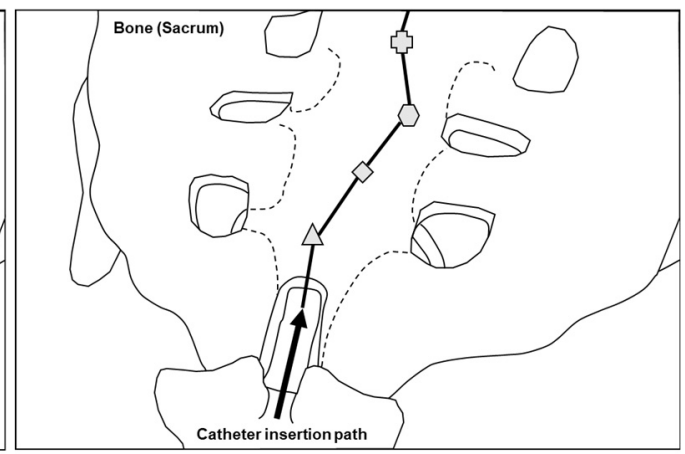

b) Path information learning step

Fig. 1. The catheter insertion path of epiduroscopy based on spatial cognitive learning

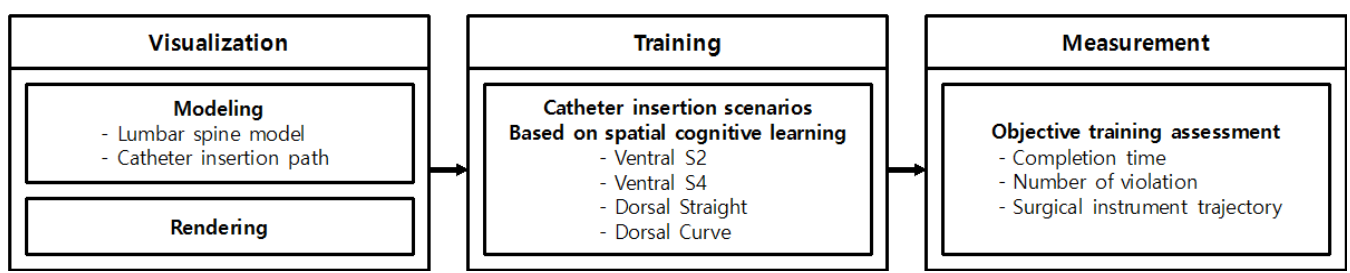

Fig. 2. The procedure of the proposed epiduroscopy training simulator based on spatial cognition learning

The visualization part models the lumbar model and catheter insertion path for training, and provides the graphic rendering. The visualization part models the training lumbar model for training based on the human body model as shown in Fig. 3(a) and the catheter insertion path as shown in Fig. 3(b). The lumbar model for training consisting of bones, disc and dura mater provides the visual feedback for the spatial cognitive learning to the trainee through graphical rendering. 
The training department provides the trainees with a basic training scenarios requiring fine catheter manipulation capability. In other words, the catheter manipulation training scenarios aim to improve the trainees' capability to manipulate the catheter inserted within human body including various nerves, lumbar bones and discs based on the spatial cognitive learning.

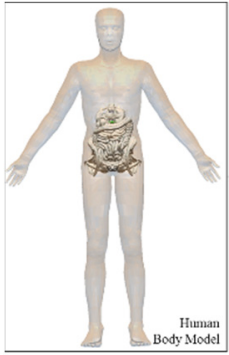

a) The lumbar models

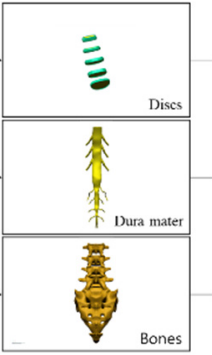

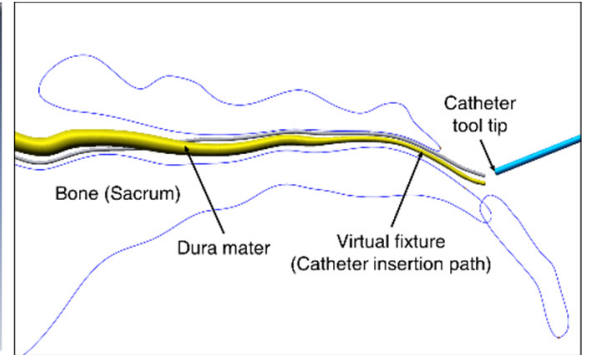

b) Catheter insertion path

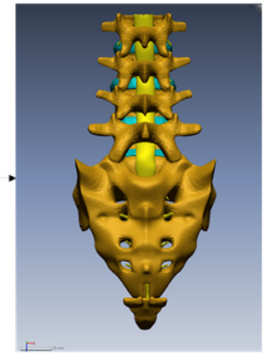

Fig. 3. Epiduroscopy models

For this, the four catheter manipulation training scenarios are selected based on the anatomical findings of the clinical expert as shown in Fig. 4. Fig. 4(a) is a catheter insertion path that enters the ventral root and then changes direction from the point between the S2-S3 nerves to the dorsal root to reach the lesion at the front of D4. Fig. 4(b) shows a catheter insertion path that enters the ventral path and detours to the dorsal root between the discs D5-S1 to reach the lesion at the front of D4. Fig. 4(c) shows a catheter insertion path that enters the dorsal root and reaches the lesion at the front of D4. Lastly, Fig. 4(d) is a catheter insertion path that enters the dorsal root and reaches a lesion on the side of $\mathrm{D} 4$.

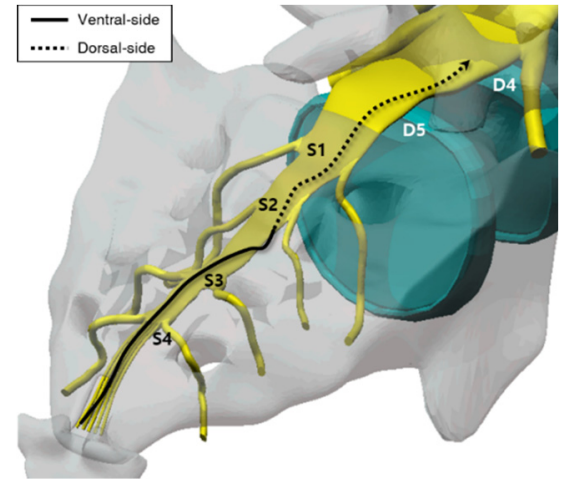

a) Ventral (S2)

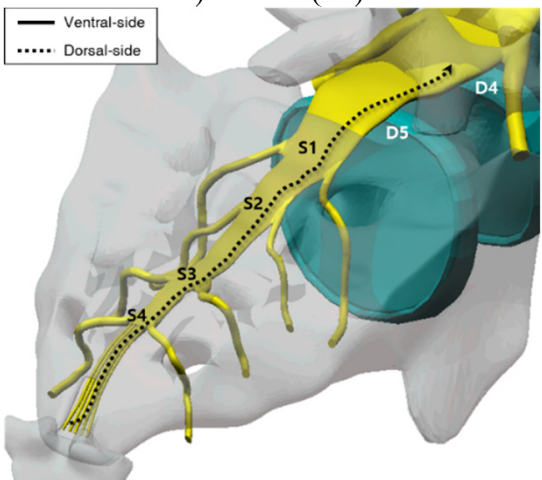

c) Dorsal (Straight)

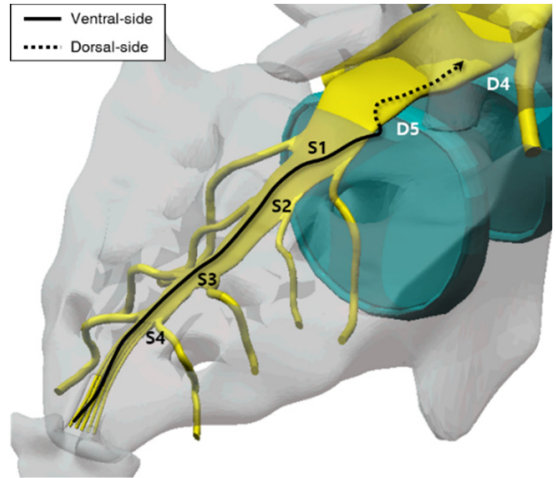

b) Ventral (S4)

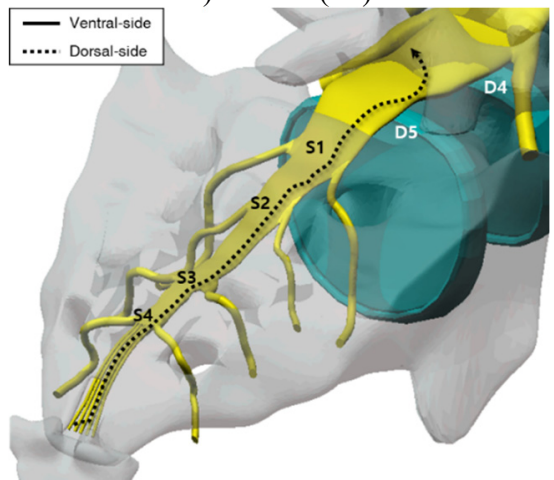

d) Dorsal (Curve)

Fig. 4. Four catheter manipulation training scenarios 
The four selected catheter manipulation training scenarios shown in Fig. 4 provide the trainees with 3D geometric information about the epidural space shown in Fig. 5. The 3D geometric information of Fig. 4 consists of the landmark information and path information corresponding the bones, discs and dura mater and catheter insertion path in Fig. 5. And it provides spatial cognitive learning through repetition of the catheter manipulation training scenarios.

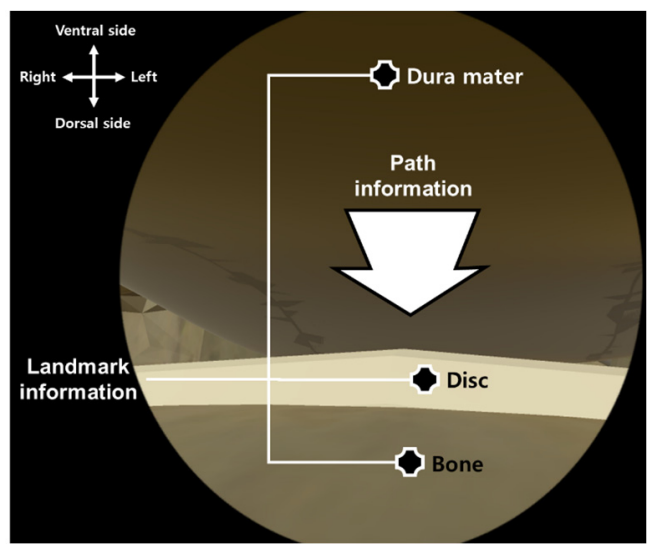

Fig. 5. 3D geometric information including landmark information (bone, disc, dura mater) and path information (catheter insertion path)

The measurement part provides the trainees with quantitative training results at the completion of training through the measurement of various data (completion time, number of violation and surgical instrument trajectory) recorded during the catheter manipulation training. It is important that the training simulator provides not only virtual environment which can mimic a real one but also an objective training assessment. The training path model for catheter manipulation is controlled through virtual fixtures installed in the epidural space.

The virtual fixture provides trainees kinesthetic feedback through collision handling and reaction force feedback, and it allows effective training of catheter manipulation [13]. In general, surgical evaluation is performed by quantifying the operation time (completion time), the mistakes occurred during training (number of violation) and the surgical instrument trajectory [14]. Therefore, the proposed training simulator uses the competition time, the number of violation and the surgical instrument trajectory as assessment factors: In other words, at the completion of epiduroscopy training, the trainees are provided with completion time, number of violation and surgical instrument trajectory as aggregated training results.

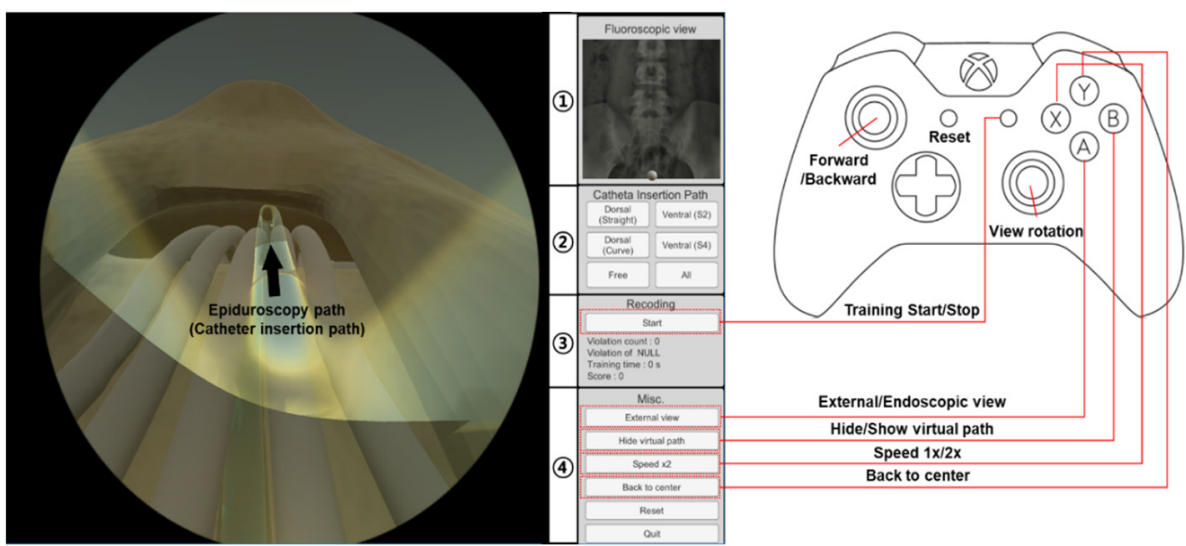

Fig. 6. Epiduroscopy training simulator developed based on spatial cognitive learning 
Fig. 6 shows the epiduroscopy training simulator developed based on the spatial cognitive learning. A game controller (Xbox pad, Microsoft, USA) is used for the interface device of the developed training simulator [15]. The developed training simulator provides couple of functions menu including a fluoroscopy view, catheter insertion path, recording and miscellaneous menus. The fluoroscopy view function ( 1 in Fig. 6) provides a view similar to the $\mathrm{C}$-arm view used in real surgery, and the catheter insertion path function (2 in Fig. 6) provides four training scenarios (ventral S2, ventral S4, dorsal straight, and dorsal curve). The recording function (3 in Fig. 6) measures and records the trainees' training data (number of violation (contact), area of violation (contact), completion time and score) in real time, and the miscellaneous menu function (4 in Fig. 6) includes virtual fixture visualization, catheter movement speed control $(1 \mathrm{x} / 2 \mathrm{x})$ and position correction at catheter deviation.

\section{Conclusions}

In this paper, an epiduroscopy training simulator based on spatial cognition learning was proposed. The proposed training simulator provided four scenarios for catheter manipulation training. The training scenarios provided trainees with 3D geometric information of epidural space to improve catheter manipulation capability inserted inside the human body, such as various nerve, lumbar and disc, based on spatial cognitive learning. Furthermore, it could provide the measured data to assess the trainees' skills objectively when the training was done.

\section{Acknowledgements}

This research was financially supported by the "Global Collaborative R\&D Program" through the Ministry of Trade, Industry and Energy (MOTIE) and Korea Institute for Advancement of Technology (KIAT).

\section{References}

[1] Roberts K. E., Bell R. L., Duffy A. J. Evolution of surgical skills training. World Journal of Gastroenterology, Vol. 12, Issue 20, 2006, p. 3219-3224.

[2] Pan J. J., Chang J., Yang X., Liang H., Zhang J. J., Qureshi T., Hickish T. Virtual reality training and assessment in laparoscopic rectum surgery. The International Journal of Medical Robotics and Computer Assisted Surgery, Vol. 11, Issue 2, 2015, p. 194-209.

[3] Moulton C. A. E., Dubrowski A., MacRae H., Graham B., Grober E., Reznick R. Teaching surgical skills: what kind of practice makes perfect: a randomized, controlled trial. Annals of Surgery, Vol. 244, Issue 3, 2006, p. 400-409.

[4] Davoudi M., Osann K., Colt H. G. Validation of two instruments to assess technical bronchoscopic skill using virtual reality simulation. Respiration, Vol. 76, Issue 1, 2008, p. 92-101.

[5] Jalink M. B., Goris J., Heineman E., Pierie J. P. E., Henk O. The effects of video games on laparoscopic simulator skills. The American Journal of Surgery, Vol. 208, Issue 1, 2014, p. 151-156.

[6] Stunt J. J., Kerkhoffs G. M. M. J., van Dijk C. N., Tuijthof G. J. M. Validation of the ArthroS virtual reality simulator for arthroscopic skills. Knee Surgery, Sports Traumatology, Arthroscopy, Vol. 23, Issue 11, 2015, p. 3436-3442.

[7] Lynch K. The Image of the City. MIT Press, Vol. 11, 1960.

[8] Hart R. A., Moore G. T. The development of spatial cognition: a review: AldineTransaction, 1973.

[9] Moore S. F., Gross S. J. Influence of critical signal regularity, stimulus event matrix, and cognitive style on vigilance performance. Journal of Experimental Psychology, Vol. 99, 1973, p. 137-139.

[10] Siegel A. W., White S. H. The development of spatial representations of large-scale environments. Advances in Child Development and Behavior, Vol. 10, 1975, p. 9-55.

[11] Kulhavy R. W., Stock W. A. How cognitive maps are learned and remembered. Annals of the Association of American Geographers, Vol. 86, Issue 1, 1996, p. 123-145.

[12] Ramloll R., Mowat D. Wayfinding in virtual environments using an interactive spatial cognitive map. IEEE in Proceedings of Information Visualisation, 2001, p. 574-583. 
[13] Ko J., Jang S. W., Kim Y. S. Development of epiduroscopy training simulator using haptic master device. IEEE 14th International Conference on Ubiquitous Robots and Ambient Intelligence (URAI), 2017, p. 542-543.

[14] Aggarwal R., Grantcharov T. P., Eriksen J. R., Blirup D., Kristiansen V. B., Funch-Jensen P., Darzi A. An evidence-based virtual reality training program for novice laparoscopic surgeons. Annals of Surgery, Vol. 244, Issue 2, 2006, p. 310-314.

[15] Vembar D., Duchowski A., Paul M., Gramopadhye A., Washburn C. Visuohaptic borescope inspection simulation training: modeling multi-point collision detection/response and evaluating skills transfer. Proceedings of Graphics Interface, 2010, p. 177-184. 